\section{Cahiers de Narratologie}

Analyse et théorie narratives

$35 \mid 2019$

Le style comme événement

\title{
Quand le texte fait événement, ou du plateau multistable
}

\section{Mathilde Vallespir}

\section{(2) OpenEdition \\ Journals}

Electronic version

URL: http://journals.openedition.org/narratologie/9404

DOI: $10.4000 /$ narratologie. 9404

ISSN: 1765-307X

Publisher

LIRCES

\section{Electronic reference}

Mathilde Vallespir, «Quand le texte fait événement, ou du plateau multistable », Cahiers de Narratologie [Online], 35 | 2019, Online since 03 September 2019, connection on 10 December 2020. URL : http:// journals.openedition.org/narratologie/9404; DOI : https://doi.org/10.4000/narratologie.9404

This text was automatically generated on 10 December 2020.

Article L.111-1 du Code de la propriété intellectuelle. 


\title{
Quand le texte fait événement, ou du plateau multistable
}

\author{
Mathilde Vallespir
}

1 Que peut apporter la notion d'événement à une stylistique? Et comment interpréter cette notion, en particulier telle qu'elle fut définie par une certaine philosophie française des années 1970 ?

2 Nous voudrions montrer la façon dont on peut articuler de telles conceptions de l'événement avec la perspective énactive de Francisco Varela, et en particulier l'expérience perceptive de multistabilité qu'il décrit dans ce cadre, afin de cerner l'effet de style comme événement multistable. Après avoir tenté de mettre en évidence les différents traits propres à la conception de l'événement développée par la philosophie du tournant des années 1970 en France, nous mesurerons en quoi cette définition peut intéresser la stylistique et autoriser la saisie du style comme événement, en caractérisant ce dernier, du fait de l'affinité entre cette définition de l'événement et la notion varélienne d'énaction, comme plateau multistable.

\section{L'événement dans la philosophie française des années 1970 : Foucault, Deleuze, Lyotard}

3 La notion d'événement a connu, dans la philosophie des années 1970, un retentissement important. On la retrouve ainsi, par exemple, chez Lyotard, Deleuze, et Foucault. Bien que définie différemment chez chacun d'eux, et à des moments légèrement différents, pour Deleuze dès 1968 dans Logique du sens, chez Lyotard, en 1971 dans Discours, figure, enfin, chez Foucault dans les années 1970, où son sens connaît une mutation (Revel 2010: 93-94'), leurs définitions partagent plusieurs caractéristiques.

4 Tout d'abord, c'est sa nature irruptive qui les caractérise. Ainsi, l'événement est pour Foucault, selon J. Revel, l'«irruption d'une singularité non nécessaire» (Revel 2010 : 96) ; pour Lyotard, la figure «fait événement linguistique, parce qu'elle est un effet de 
décharge provenant d'un autre ordre " (Lyotard 1985 [1971] : 146). Enfin, l'événement est tenu chez Deleuze, dans une lignée stoïcienne, pour "disjonction exclusive", propre à opérer une «scission interne de la temporalité » (Zourabichvili 2003 : 40). Par ailleurs, comme chez Foucault, il n'est pas réductible au logos. Ainsi, si pour Foucault, l'événement est réfractaire à un principe de causalité linéaire, étant le "produit de causalités multiples et complexes qui s'entrecroisent » (Revel 2010 : 96), de même qu'il se dérobe à toute approche structurale ${ }^{2}$, chez Deleuze, il ne peut être réduit à la proposition, et il ne peut relever du « discours » chez Lyotard, qui est le lieu de la mise en œuvre d'un langage répondant aux lois du logos, à son ordre ${ }^{3}$. Pour autant, chez les trois philosophes, cet événement advient bien au sein du langage. La figure est rhétorique, donc avant tout élaborée à partir du langage chez Lyotard ${ }^{4}$, de même pour Deleuze, pour lequel l'événement fait l'objet d'une ressaisie dans le langage ${ }^{5}$, quand pour Foucault, l'événement est «événement discursif » (Foucault 1994 : 467) : dans la méthode archéologique puis généalogique, c'est en effet depuis les discours que les événements sont évalués. Autrement dit, ce sont les discours qui font événement.

Une telle caractéristique en engage une troisième: l'événement est toujours défini comme contradictoire ou paradoxal. Ainsi, la tension contradictoire tient pour Lyotard, comme on vient de le voir, à ce que l'événement figural advient dans le langage mais constitue une force irruptive qui exclut l'ordre de ce dernier ${ }^{6}$. Pour Foucault, elle réside en ce que, tout en refusant de soumettre l'événement au déterminisme causal, il est pour lui en même temps «exclu de penser l'événement comme dehors, ou comme excédence radicale par rapport à toute causalité ${ }^{7}$ [...]. » Pour Deleuze, l'événement relève du langage en ce que ce dernier en effectue la synthèse disjonctive, tout en étant également " "attribut" du monde et de ses états de choses " (Zourabichvili $2003: 37$ ). Ce que F. Zourabichvili reformule ainsi : "Selon un [...] paradoxe, l'événement est ce qui du monde ne subsiste comme tel qu'en s'enveloppant dans le langage, qu'il rend dès lors possible » (Zourabichvili $2003: 10$ ). C'est également eu égard au temps entendu comme donné chronologique que l'événement est paradoxal et contradictoire. Ainsi, il implique selon Lyotard, comme le lapsus ou l'orgasme, "la "relation" de deux "états" hétérogènes et cependant jouxtés dans une anachronie irréversible» (Lyotard 1985 [1971]: 137). De même, pour Deleuze, comme le souligne F. Zourabichvili, «l'événement se donn[e] dans l'étrange station d'un encore-là-et-déjà-passé, encore-à-venir-et-déjà-là » (Zourabichvili $2003: 37$ ). Opérant, comme on l'a vu, une "disjonction exclusive", scission interne de la temporalité, il s'inscrit dans le temps tout en étant hors de lui. Deleuze décrit ainsi l'événement qu'est le présent de l'acteur comme « le plus étroit, le plus resserré, le plus instantané, le plus ponctuel, point sur une ligne droite qui ne cesse de diviser la ligne, et de se diviser lui-même en passé-futur » (Deleuze 1968 : 176). De sorte que l'événement est propre à défaire toutes les dichotomies : s'il est dans le langage et hors de lui, c'est parce qu'il « est [donc] des deux côtés à la fois, comme ce qui, dans le langage, se distingue de la proposition, et ce qui, dans le monde, se distingue des états de choses" (Zourabichvili 2003 :37-38). De même, l'événement défait la dichotomie du privé et du collectif : «il n'y a pas d'événements privés, et d'autres collectifs ; pas plus qu'il n'y a de l'individuel et de l'universel, des particularités et des généralités. Tout est singulier, et par là collectif et privé à la fois, particulier et général, ni individuel ni collectif » (Deleuze $1968: 178$ ).

6 C'est donc à partir de la structure logique de la contradiction que se définit l'événement, ni hors ni dans le langage, ni hors ni dans l'histoire et le temps, ni privé ni collectif; et ce schème logique, par lequel toute définition de l'événement passe, 
répond à sa situation particulière eu égard au langage chargé d'en rendre compte. Il ne peut ainsi que se situer sur cette «frange de la logique formelle ( Derrida 1967 : 68) réfractaire à la catégorisation qu'opère le langage.

7 Parmi ces pensées de l'événement, celle qui est la plus proche de notre objet semble être celle de Lyotard dans Discours, figure. En effet, l'événement visé dans cette œuvre est l'événement que constitue la figure dans le discours, de sorte que la notion est déjà rapportée au champ de l'esthétique et de la rhétoricité. Sa saisie de l'événement, outre les traits précédemment évoqués et communs aux autres philosophes cités, implique également un caractère dynamique : ainsi, la figure qui « fait événement linguistique ", "ne peut être saisie qu'en termes énergétiques"; elle relève de la force, laquelle s'oppose au système de la langue (Lyotard 1985 [1971] : 146). Cette force, qui est celle de la pulsion, fait violence au logos et à ses structures ${ }^{8}$ pour faire advenir la vérité, qui se situe en-deçà ou au-delà de ce logos; elle est «blessure » faite aux mots (Lyotard 1985 [1971] : 327). C'est pourquoi, selon Lyotard, l'événement se dérobe à l'intentionalité phénoménologique : il ne relève pas de la connaissance, de sorte que l'intentionalité ne peut le saisir. Lyotard met ainsi en valeur les limites de la compréhension de Cézanne par Merleau-Ponty. Et le concept central apporté par la phénoménologie pour penser l'événement serait non l'intentionalité mais la passivité :

Que la montagne Sainte Victoire cesse d'être un objet de vue pour devenir un événement dans le champ visuel, c'est cela que Cézanne désire, c'est cela que le phénoménologue espère comprendre, et que je crois qu'il ne peut pas comprendre. Son concept ultime, son concept le plus fin pour s'emparer de l'événementialité du donné, ce n'est certes pas l'intentionalité, c'est la passivité. (Lyotard 1985 : 21)

8 Cette passivité comme laisser-être, «désaisissement» (Lyotard, 1971: 23), proche de l'écoute flottante pratiquée par la psychanalyse (Lyotard, 1971:381), est la seule voie possible pour « s'emparer de l'événementialité du donné » (Lyotard, 1971 : 21). Lyotard, qui fait ici référence à l'événement visuel, propose ailleurs plusieurs exemples de métaphores, dont «je te musique» de Pichette, comme propre à «faire événement linguistique " (Lyotard 1971:1469). Cet événement, qui ne peut donc se donner que via une réception passive et soustraite à l'intentionalité de la connaissance, constitue le lieu d'avénement de la véritée ${ }^{10}$.

Nous voudrions proposer de penser l'événément stylistique à partir de la conception que l'on vient de déployer de l'événement, tant à partir des traits définitionnels communs précédemment mis en lumière (irruptif, dynamique, antistructural, contradictoire, et propre à trouver sa source dans le langage tout en l'outrepassant), qu'à partir de la notion d'événement figural chez Lyotard, qui a donc pour caractéristique supplémentaire de se manifester dans le logos en faisant violence à ses structures, et se joue dans une relation que l'on peut saisir comme passivité non intentionnelle. Pour ce faire, deux aspects restent à préciser ici.

D'une part, il restera à penser la relation de la figure selon Lyotard au style comme effet produit sur le récepteur, figure et style ne pouvant être co-extensifs l'un à l'autre, ce sur quoi nous reviendrons en fin de développement.

D'autre part, si Lyotard donne les caractéristiques précises d'un tel événement figural, en l'articulant à un large champ philosophique, mais aussi linguistique et logique ${ }^{11}$, toutefois, ne se trouve pas décrit en soi ce mode de cognition non intentionnelle comme tel, propre à autoriser l'événement. Une telle description supposerait de 
recourir à d'autres outils, ceux de la psychologie cognitive par exemple, encore non développés dans les années d'écriture et de parution de Discours, figure.

Nous souhaitons donc à présent tenter d'opérer une telle extension ou articulation de la pensée de l'événement des philosophes de ces années 1970 et de Lyotard à une théorie autorisant une telle représentation de l'agencement cognitif propre à laisser place à cet événement stylistique : celle de Francisco Varela. Cet événement stylistique, nous le redéfinirons comme émergence ou énaction d'un agencement de réception multistable.

\section{Événement, énaction et multistabilité}

13 Si a priori il semble y avoir un saut disciplinaire entre la philosophie des années 1970 et la neurobiologie de F. Varela, ce saut n'est qu'apparent. Le neurobiologiste a en effet édifié une théorie qu'il nomme lui-même " neuro-phénoménologie ", et eut ce scrupule de donner un cadre philosophique aux représentations du fonctionnement neuronal qu'il élabore, trouvé dans la phénoménologie husserlienne (Varela 2002 : 342-343). Or, c'est à partir (et pour partie contre) la phénoménologie, philosophie régnante dans la France de ces années 1970 que Lyotard construit sa conception de l'événement figural, comme on l'a vu supra. La phénoménologie (même s'il s'agit pour Varela de Husserl, et pour Lyotard de Merleau-Ponty) constitue donc un cadre commun à ces deux pensées. Au-delà, l'événement répond par bien des points à la notion d'énaction varelienne.

Comme nous l'avons déjà présentée (Vallespir, 2012), la notion d'énaction, « de l'anglais to enact : susciter, faire advenir, faire émerger ", "loin d'être la représentation d'un monde prédonné, est l'avènement conjoint d'un monde et d'un esprit à partir de l'histoire des diverses actions qu'accomplit un être dans le monde » (Varela, Thomson, Rosch 1993: 35). L'énaction semble donc partager avec l'événement son caractère irruptif ainsi que dynamique, la nature conjointe de la conscience énactante et de son environnement énacté supposant une dynamique de redétermination constante. On a ailleurs montré la possibilité de concevoir l'agencement de réception esthétique comme énaction, lecteur ou récepteur et texte ou œuvre perçue s'énactant l'un l'autre dans un agencement défini comme énaction mutuelle (Vallespir, 2012: 93). L'expérience de réception n'est alors plus conçue comme telle, mais comme coconditionnement dynamique du texte et du lecteur ${ }^{12}$. Dans un tel cadre, l'événement stylistique supposerait la cristallisation particulière d'un co-conditionnement du lecteur et du texte propre à fédérer les autres caractéristiques attribuées à l'événement: il faudrait que le texte se mette à fonctionner de manière à déterminer un mode de perception qui soit en rupture avec le mode de déchiffrement du sens habituel. Cet événement stylistique, c'est dans un agencement multistable tel que défini par Varela qu'on peut le trouver.

Comme nous l'avons montré ailleurs (Vallespir 2016, 2017a, 2017b 2019, à paraitre ${ }^{13}$ ), la multistabilité est une notion empruntée à la biologie par Varela afin de rendre compte de la conscience du temps. L'intérêt d'un tel emprunt réside dans le fait que, contrairement à une conception issue de la mécanique, où la stabilité constitue la norme, en biologie, c'est l'instabilité qui l'est (Varela, 2002:366-367). La multistabilité est donc une instabilité instaurée comme norme. Varela applique cette notion à la perception, en donnant plusieurs exemples de multistabilité perceptive. On connait le dessin du canard-lapin rendu célèbre par J. Jastrow, que l'on peut identifier comme la 
tête de l'un ou de l'autre animal. Varela donne d'autres exemples de ces ambiguïtés perceptives, dont une pyramide perceptible comme couloir, ou une série d'images où se trouve répétée et imperceptiblement modifiée la même représentation, un visage d'homme se muant en corps de femme portant un enfant (Varela, 2002: 365). Ces expériences perceptives, qu'il mobilise pour faire valoir la conscience de la durée, sont analysées par le neurobiologiste comme des expériences de multistabilité: la perception y oscille ainsi entre ce que Varela appelle des «bassins d'attraction stables " (Varela, 2002 : 366) - pour le canard de Jastrow, le canard et le lapin - sans forcément déboucher sur la stabilisation de la perception sur l'un de ces bassins. Cette multistabilité érigée en norme perceptive nous a semblé productive pour rendre compte de la spécificité de certaines figures (la métaphore, l'ironie), ou de constructions textuelles (Vallespir à paraître).

Une telle multistabilité, quand elle advient au sein de la lecture, nous paraît propre à y faire événement. C'est ce que nous souhaitons montrer en reprenant l'exemple que Lyotard emprunte à $\mathrm{H}$. Pichette pour mettre en évidence le fonctionnement de l'événement linguistique (Lyotard $\left.1985: 144-146^{14}\right)$, «je [...] te musique » (Pichette : 56), et en en rendant compte à l'aune de la notion de multistabilité.

\section{" Je te musique », événement multistable}

L'expression "je te musique» suppose une double impertinence, syntaxique et sémantique. Le nom "musique " étant situé après deux pronoms préclitiques ou conjoints atones, on attendrait un verbe à sa place. Cette première impertinence syntaxique suppose déjà une multistabilité, partagée entre deux bassins d'attraction stable: un dans lequel le nom serait lu comme tel, ce qui par conséquent bloque la dimension processuelle attendue dans un verbe, à laquelle le lecteur s'était préparé du fait de la présence des deux pronoms. Est alors en même temps bloquée la lecture, car les pronoms ne peuvent trouver interprétation. Un autre bassin se dessine autour de ce qu'invite à lire la présence de ces deux pronoms, et qui est autorisé par la finale en -e de «musique " analogique de la finale des verbes en -er à la première personne. "Musique » apparait alors comme forme fléchie d'un hypothétique verbe musiquer. Même si la lecture est infléchie sensiblement par le contexte vers cette seconde interprétation ${ }^{15}$, elle oscille néanmoins entre ces deux bassins d'attraction stable sans pouvoir s'arrêter à l'un ou l'autre.

Le second autorise toutefois à poursuivre l'interprétation. On l'a vu, la présence des deux pronoms programme une structure actantielle à deux actants, faisant de musiquer un verbe transitif direct. Or, l'expression la plus proche impliquant le nom « musique » mais supposant un procès est « je fais de la musique », qui exclut un actant complément direct. La lecture en est d'autant complexifiée, plus encore du fait que la structure actantielle du verbe, très courante et propre à des verbes très divers (je te parle, je te vois, je t'aime, je te tue), ne programme aucun procès qui pourrait constituer un métaphorisé de prédilection, et donc un bassin d'attraction métaphorique stable évident. Sans contexte, le lecteur peut donc ici hésiter entre deux types de réduction de cette impertinence: soit le verbe néologique musiquer est à prendre en ce qui serait son sens le plus concret. Alors «je te musique " pourrait signifier "je te transforme en musique", "je fais de toi de la musique», "te» désignant alors soit l'allocutaire (on serait alors dans un monde contrefactuel où l'on 
peut transformer quelqu'un en musique, comme dans les contes on transforme les hommes en lapin ou en licorne ; on peut alors penser à la photographie de Man Ray, "Le Violon d'Ingres ${ }^{16}$ ", où l'artiste représente son modèle comme un instrument), soit, de manière métonymique cette fois, l'image mentale ou la représentation que le locuteur a de l'allocutaire (et non la personne physique elle-même). L'expression pourrait alors être paraphrasée par « je fais une musique sur toi », et mise en série avec l'utilisation transitive directe avec un complément d'objet humain du verbe chanter (comme dans " Dante a chanté Béatrice »). D'un point de vue sémantique, on aurait ici un autre bassin d'attraction stable, déjà décliné en deux possibilités voisines. Il y en aurait encore un autre, dans le cas où ce qui est envisagé dans « je te musique ", c'est une action qui ressemble à la musique, qui comporte les traits prêtés par le locuteur à la musique, l'expression pouvant alors signifier « je te fais quelque chose qui pour moi a une ou des caractéristiques attribuées par moi à la musique ». " Musique " aurait alors un sens métaphorique, dont les contours sont faiblement déterminés, compte tenu de l'actualisation des possibles ou sèmes afférents véhiculés par le terme, si ce n'est sa nature assez clairement valorisante. On peut penser que ce "musique » désigne potentiellement tout procès supposant ces sèmes afférents (douceur, délicatesse, subtilité, charme, beauté), ce qui laisse donc un large champ de possibles couvrant potentiellement toute la gamme des sentiments et actes amoureux ou érotiques, et ce que le contexte confirme: rappelons qu'il s'agit d'un duo d'amour entre le poète et l'amoureuse. L'expression pourrait alors signifier par exemple « je te caresse/t'enlace/ t'embrasse/te désire », mais ce ne sont là que quelques possibilités, et aucune n'exclut les autres. Les différents bassins d'attraction stables que l'on a identifiés, liés à la double impertinence syntaxique et sémantique que présente le tour, sont donc au moins au nombre de trois, selon que «musique » est tenu pour nom ou verbe, et dans ce second cas, qu'il est pris en un sens plus ou moins concret. On a vu de surcroît que ces bassins d'attraction stables entre lesquels hésite et oscille la perception du texte, supposent à leur tour une grande plasticité sémantique, aucune interprétation univoque ou traduction n'étant possible. La compréhension du passage repose donc sur un va-et-vient ou une co-présence de cet ensemble de possibilités interprétatives, ou plutôt le parcours de cet ensemble. Sans spécification contextuelle, cette instabilité demeure totale, et la perception ne peut s'arrêter sur une lecture possible, elle est contrainte d'errer entre les bassins d'attraction ainsi envisagés. Et même en présence $\mathrm{du}$ contexte, la lecture demeure instable, et continue de se jouer dans cette oscillation entre les trois bassins d'attraction.

La multistabilité à laquelle donne lieu ce passage est donc particulièrement dense ; et, bien que l'ensemble de ce duo présente de nombreuses figures, en particulier de métaphores, et soit donc particulièrement multistable, ce «je te [...] musique », dans son microcontexte, constitue un lieu de haute multistabilité. Comme, de plus, cette multistabilité ne trouve pas de stabilisation, on pourrait ici parler de plateau de multistabilité, dans le sens originel que peut avoir ce terme emprunté par Deleuze à l'anthropologue G. Bateson, et dont Deleuze et Guattari rappellent la définition : « une région continue d'intensités, vibrant sur elle-même, et qui se développe en évitant toute orientation sur un point culminant ou vers une fin extérieure " (Deleuze et Guattari $\left.1980: 32^{17}\right)$.

20 Dans cette mesure, ce haut degré de multistabilité, malgré sa configuration particulière de plateau et malgré un contexte où la présence d'autres irruptions constituées par les autres figures donne lieu à une habituation du lecteur et à une sorte d'apprivoisement 
des figures, ou plutôt, d'apprivoisement à la multistabilité qu'elle engendrent, constitue bien une rupture, une irruption dans le texte. On comprend dès lors à quel point l'analyse multistable a vocation à saisir la dimension dynamique de la figure, cette dynamique résidant dans une oscillation entre différentes lectures possibles qui ne sont en elles-mêmes jamais véritablement arrêtées et menées à leur terme, sinon dans le geste analytique. Pour autant, on peut souligner que cette multistabilité peut être analysée en termes de contradiction, dans la mesure où les différents bassins d'attraction, analysés en termes logiques et figuraux, peuvent mener à des résultats interprétatifs opposés et inconciliables: ainsi, que "musique " soit interprété comme nom ou verbe, qu'il soit métaphorique ou non métaphorique dans "je te musique " sont des interprétations inconciliables, contradictoires du point de vue syntaxique et sémantique, et pourtant, cumulées dans la lecture multistable. La configuration « je te musique ", qui relève bien du langage, suppose donc un fonctionnement sémiotique complexe de ce dernier, supposant de remettre en cause l'analyse structurale, saussurienne, du langage comme association stable d'un signifiant à un signifié. Ici, la structure signifiante reçoit tour à tour des interprétations sémantiques différentes qui se groupent autour des bassins d'attraction déterminés plus haut ; c'est donc à dire qu'à un signifiant correspondent plusieurs signifiés. Mais ce serait encore prétendre à une association stable entre signifiant et signifié autour d'un bassin d'attraction, ce qui n'est pas le cas: en effet, on l'a vu, l'interprétation métaphorique de «musique » ne peut être arrêtée aussi nettement que certaines métaphores peuvent l'être, telles que "Achille est un lion» - dont il faut rappeler qu'elle-même n'est pas stable non plus (Vallespir 2017b: 49018). Chaque bassin suppose une sorte de halo de signifiés qui ne peuvent être distingués les uns des autres. Ce plateau multistable répond donc à un mode de fonctionnement sémiotique du langage qui, sans en être un au-delà, puisqu'il est fabriqué dans le langage, suppose une remise en question de l'organisation catégorielle qu'il instaure: que ce soit en effet d'un point de vue syntaxique ou sémantique, et dans cette dernière catégorie, au sein d'un bassin d'attraction ou entre les différentes solutions qu'il suppose, la stabilité catégorielle induite par la correspondance entre signifiant et signifié est largement mise à mal ${ }^{19}$. Enfin, si l'on ne peut parler de passivité totale de la part du lecteur, l'activité herméneutique n'est pas celle de l'analyste ou du lecteur "d'archive» (Molinié 1998: 13620). Ceci induit également, outre le fait de moindrement pousser les différentes interprétations possibles et de ne pouvoir trancher entre elles, de se laisser davantage altérer par les configurations multistables, et donc, de pratiquer un certain «lâcher-prise " par rapport à un régime de compréhension du texte plus fréquent, valable dans des contextes de moindre densité figurale par exemple.

21 Caractère dynamique, contradictoire, phénomène langagier tout en dérogeant au fonctionnement structural du langage et propre à faire jouer ses structures catégorielles, supposant des conditions de perception où la cognition s'effectue par un lâcher-prise, et donc une certaine passivité: on reconnaît là l'ensemble des caractéristiques attribuées à l'événement par la philosophie des années 1970 et par Lyotard. On a donc redécrit ce que Lyotard avait choisi pour exemple de l'événement comme plateau multistable. Or, cette description répond, par sa configuration même, que Lyotard n'avait pas comme telle mise à nu, à la définition de l'événement donnée par cette philosophie des années 1970. Il ne s'agit pas ici, en un geste tautologique, de revalider ce qui avait déjà été donné par Lyotard, ou de valider notre description en la rabattant sur l'analyse du philosophe. Mais l'agencement d'énaction dans lequel 
s'inscrit « je te musique ", que l'on a décrit comme plateau multistable, comporte à son tour, par sa multistabilité même, les caractéristiques de l'événement. La notion d'événement s'en trouve ainsi dotée d'une nouvelle extension possible - mais possible seulement : car l'agencement énactif impliquant «je te musique », en tant que plateau multistable, n'est pas co-extensif à tout événement, mais en constitue une forme possible. En effet, d'une part, la multistabilité peut ne pas faire événement en ce qu'elle peut être tenue pour mode standard de lecture. Comme le soulignent Nathalie Blanc et Denis Brouillet, « [1]a représentation cognitive est [...] fondamentalement dynamique et instable» (Blanc \& Brouillet 2005 : 131). La lecture en temps réel repose en effet sur l'aptitude à sélectionner les informations, c'est-à-dire à "inhiber » les hypothèses interprétatives erronées (Blanc \& Brouillet 2005:147 et sq.), ce qui suppose une incessante réévaluation des hypothèses de lecture. En tant que norme perceptive, la multistabilité ne peut donc pas être irruptive. En revanche, quand elle se mue en plateau multistable, où la perception ne peut se réduire à un des bassins d'attraction et que ce bassin demeure lui-même si plastique que cette multistabilité enfreint la catégorisation sans pouvoir in fine y être réduite, alors, la multistabilité peut regagner le caractère irruptif de l'événement, même si cette irruptivité a une forme paradoxale de plateau.

Nous avons ici analysé cette multistabilité dans le cas d'une figure complexe, affectant à la fois la syntaxe et la sémantique, comme nous l'avions fait précédemment pour la métaphore et l'ironie. Mais cet événement multistable passe les limites de la figuralité, et peut être à l'œuvre à la faveur d'autres configurations textuelles. On a ainsi mis en valeur la façon dont elle se jouait dans La Chevelure de Bérénice de Claude Simon entre des blocs représentationnels entre lesquels le lecteur hésitait (Vallespir à paraître). Bien que le caractère événementiel soit dans cette œuvre récurrent, il n'en conserve pas moins sa nature; on peut d'ailleurs déceler dans cette récurrence événementielle une des raisons de la densité esthétique de l'œuvre. On pourrait également mettre en valeur une telle multistabilité dans le cadre du discours philosophique. Dans ce cas, une structure logique telle que la contradiction, ainsi qu'on la trouve chez Levinas ou Blanchot, nous paraît constituer un site possible de multistabilité, voire de plateau multistable. La multistabilité se jouerait ici entre deux bassins d'attraction constitués par deux propositions contradictoires (c'est-à-dire opposées et en relation de disjonction exclusive) en même temps énoncées. Cette oscillation entre les deux bassins logiques ne pouvant trouver de résolution, elle est donc propre à faire plateau et par là événement.

La multistabilité et la possibilité événementielle qu'elle comporte excèdent donc le champ de la seule figuralité, mais aussi les limites propres aux genres et aux types de discours.

\section{Conclusion}

Nous avons donc essayé, après avoir proposé une redéfinition de la notion d'événement à l'aune d'une certaine philosophie française des années 1970, de montrer la façon dont la notion de multistabilité, empruntée à Francisco Varela, pouvait être féconde pour penser un certain type de configurations linguistiques propres à "faire événement " dans la lecture. Cet événement prend la forme d'un plateau multistable qui est tel du fait de l'absence d'inhibition d'hypothèses de lecture: si, dans une lecture qu'on 
qualifierait de standard (mais en est-il jamais ?), les hypothèses de lecture paraissant erronées se trouvent inhibées, comme on l'a vu supra, on peut ici soumettre l'hypothèse que dans le cas de plateau multistable, cette inhibition se révèle longue, elle-même instable, voire impossible, et que des lectures opposées se cumulent dans la mesure où il est impossible de déterminer de lecture préférentielle, aucune hypothèse ne pouvant être jugée erronée et donc aucune ne pouvant être à ce titre inhibée.

On a ailleurs posé l'articulation entre style, fait de langue et effet de style à l'aune de l'énaction (Vallespir 2014:30-31). Ainsi, dans cette perspective, où texte et lecteur se conditionnent réciproquement, le fait de langue correspond à la «spécification du texte par le lecteur", l'effet de style à la "spécification du sujet par le texte ou « altération du sujet lecteur par le texte » (Vallespir 2014 : 30). L'événement multistable peut dans ce cadre être tenu pour un effet de style conditionné par un fait de lague qui se définit par sa saillance particulière, laquelle sature l'attention perceptive du lecteur. Cette saillance prend la forme de ce co-conditionnement entre lecteur et texte où la perception du lecteur se fait plateau de multistabilité. Ces événements multistables, qui font émerger certaines configurations stylistiques, constituent des moments d'intuition du style sans lesquels aucune stylistique, aussi objective qu'elle se prétende, ne peut se construire.

Une telle conception de l'événement stylistique multistable comporte plusieurs présupposés. Elle suppose tout d'abord de considérer la lecture comme une expérience, et par là, de prendre en compte sa temporalité, son déroulement, sa nature " on line ». La notion d'événement énactif empruntée à Varela suppose également que l'on considère non pas seulement l'effet produit sur le lecteur, ou le texte en lui-même, mais l'agencement impliquant à la fois le lecteur et le texte, et le conditionnement réciproque de l'un et de l'autre au sein de cet agencement. Une telle conception englobante de l'événement stylistique implique de pouvoir penser l'ensemble complexe des conditions qui y donnent lieu, et l'interaction des paramètres l'impliquant. Ainsi, ce plateau multistable résulte d'un ensemble des déterminations historiques du corpus et de la lecture, et également de la façon dont elles s'interdéterminent. Dans tous les cas étudiés, la multistabilité est liée à des œuvres relevant d'une certaine modernité, et répondant à une lecture qui leur est un peu postérieure, qui a pris acte à la fois des théories de la complexité et des pensées anti-structurales et anti-systémiques des années 1970. C'est l'interconditionnement de ces éléments qui donne lieu à une telle approche; on pourrait penser que les événements multistables seraient moins nombreux dans un corpus classique, chez Racine ou Bossuet, ou du moins qu'ils prendraient une autre forme chez Cyrano de Bergerac (on pourrait peut-être les retrouver dans le jeu des impossibilia par exemple, de même que dans l'ironie et le jeu parodique imposé aux patrons rhétoriques). L'agencement théorique multistable permet ainsi de prendre en compte ces éléments et de rendre compte du résultat de leur interaction. Outre cette prise en compte des conditions historiques de production de l'œuvre comme de sa réception, une telle conception peut permettre de rendre compte d'une certaine gradualité : on peut envisager des événements stylistiques plus ou moins multistables, plus ou moins événementiels donc, et ordonner les événements selon leur plus ou moindre saillance, ou plutôt, rendre compte de types d'événements différents à la saillance se manifestant différemment. Ainsi, la saillance de «je te musique ", dans son contexte, entraîné par toutes les métaphores qui l'entourent et l'emportent, est moindre que celle d'une métaphore advenant en contexte 
moindrement métaphorique. De même, la multistabilité de l'ironie d'un texte pourra être moindre que celle d'un autre. Et on pourra considérer que la multistabilité engendrée par la métaphore " Achille est un lion » est moindre que l'ironie d'un poème de Michaux. Une telle conception de l'effet de style comme événement multistable peut autoriser une description historicisée et nuancée des phénomènes stylistiques.

Elle permet aussi de dépasser la possible contradiction existant entre la singularité du style et la continuité des structures linguistiques. En effet, l'événement multistable est, on l'a vu, irruptif; il suppose une rupture par rapport à l'entour textuel, mais aussi par rapport à l'ensemble des structures déjà rencontrées par le lecteur, et en cela, constitue une configuration inédite et singulière pour ce dernier. En même temps, la gradualité que nous venons de souligner, due au fait que cet événement répond au modèle du plateau, implique qu'il n'y ait pas de rupture entre un régime de lecture normalement multistable, où la multistabilité se trouve constamment réduite par l'élimination des hypothèses tenues pour erronées, et le régime de plateau multistable propre à l'événement stylistique, où la densité de multistabilité implique l'impossibilité de réduire les hypothèses de lecture. S'il y a certes un seuil, il n'est en revanche pas de différence de nature entre le fait de style et l'effet qu'il provoque d'une part, et le reste de la langue d'autre part. En cela, ce modèle peut permettre de conjoindre perspective continuiste et esthétique, descriptive historique et esthétique, de même qu'une stylistique des patrons et de la singularité.

Enfin, envisager l'événement stylistique comme un plateau de multistabilité suppose une conception antiphallocentrique (antiphallogocentrique, aurait dit Derrida) de l'événement esthétique. En effet, le modèle du plateau emprunté à $G$. Bateson par Deleuze et Guattari est un lieu instable de non orientation, de désorientation du fait de la tension entre plusieurs possibles lectures, mais non orienté vers une résolution. L'événement multistable en tant que lieu d'indécision, d'hésitation et de suspens, défait l'irruptivité de l'événement de toute perspective phallocentrique et lui ouvre la possibilité d'être conçu de manière discontinue mais non binaire.

\section{BIBLIOGRAPHY}

Blanc, Nathalie \& Brouillet, Denis (2005) : Comprendre un texte. L'évaluation des processus cognitifs, Paris, Éditions In Press.

Deleuze, Gilles (1968) : Logique du sens, Paris, Minuit.

Deleuze, Gilles \& Guattari, Félix (1980) : Mille plateaux, Paris, Minuit.

Derrida, Jacques (1967) : Linguistique et grammatologie, Paris, Minuit.

Foucault, Michel (1994) : Dits et écrits 1954-1988, III, 1976-1979, Paris, Gallimard.

Gualandi, Alberto (2017) : « Voix, corps, langage. Réflexions quasi-psychanalytiques autour de J.-F. Lyotard », in F. Fruteau de Laclos \& C. Énaudeau (dir.), Lyotard et le langage, Paris, Klincksieck, p. 277-290. 
Klinkenberg, Jean-Marie (2000) : Précis de sémiotique générale, Paris, Le Seuil.

Lyotard, Jean-François (1985 [ $\left.\left.{ }^{1} 1971\right]\right)$ : Discours, figure, Paris, Klincksieck.

Molinié, Georges (1998) : Sémiostylistique. L'effet de l'art. Paris, Klincksieck.

Pichette, Henri (1969 [19198]) : Les Épiphanies, Paris, Gallimard.

Revel, Judith (2002) : Le Vocabulaire de Foucault. Paris, Ellipses.

- (2010) : Une pensée du discontinu, Paris, Mille et une nuits.

Vallespir, Mathilde (2012) : «Pour une sémiotique de l'énaction, ou comment réduire la violence du logos analytique ", in L. Kurts-Wöste, M. Vallespir, M.-A. Watine (dir.), La Violence du logos : entre sciences du texte, philosophie et littérature, Paris, Garnier, p. 81-100.

- (2014) : « Pour une herméneutique de l'énaction : à partir d'un fait de langue métaphorique complexe », in L. Himy-Pieri (dir.), Le Style découpeur de réel, Rennes, Presses Universitaires de Rennes.

- (2016) : «Stylistique littéraire, musique et "veille sémiotique" : l'exemple de l'ironie ", Musurgia 2016/1-2-3, Vol. XXIII, p. 11-25.

- (2017a) : «Énaction et pragmatique du discours : l'ironie comme processus discursif multistable », Signifiances (Signifying), 1 (1), 91-100.

- (2017b) : "Simplicité et complexité, ou comment penser le simple à la lumière du complexe », in S. Jollin-Bertocchi, L. Kurts-Wöste, Anne-Marie Paillet \& Claire Stolz, La Simplicité, manifestations et enjeux culturels, Paris, Champion, p. 483-492.

- (à paraître) : «Complexité perceptive chez Claude Simon : instabilité référentielle et multistabilité dans La Chevelure de Bérénice », in M.-A. Watine, I. Yocaris \& D. Zemmour (dir.), Claude Simon. Une expérience de la complexité, Paris, Classiques Garnier, coll. « Rencontres » Varela, Francisco J. (2002) : «Le présent spécieux : une neurophénoménologie de la conscience du temps ", in J. Petitot, F. J. Varela, B. Pachoud, J.-M. Roy, Naturaliser la phénoménologie, essai sur la phénoménologie contemporaine et les sciences cognitives, Paris, CNRS éditions, chapitre 8, p. 341-406. Varela, Francisco, Thomson, Evan \& Rosch, Eleanor (1993) : L'inscription corporelle de l'esprit. Sciences cognitives et expérience humaine, Paris, Seuil.

Zourabichvili, François (2003) : Le Vocabulaire de Deleuze, Paris, Ellipses.

\section{NOTES}

1. « [...] Par événement, Foucault entend plusieurs choses. L'événement, c'est d'abord, de manière tout d'abord négative, un fait dont certaines analyses historiques se contentent de fournir la pure description. [...] Cependant, dans un deuxième temps, la notion d'événement commence à apparaître progressivement chez Foucault, de manière positive, comme une cristallisation de déterminations historiques complexes qu'il oppose à l'idée de structure ».

2. Voir M. Foucault (1994 : 468, cité par Revel 2010 : 95) : « Ni la logique du sens, ni la logique de la structure ne sont pertinentes pour ce type de recherche ». Voir aussi «Entretien avec M. F.», dans A. Fontana et P. Pasquino, Microfisica del poterer : interventi politici, repris dans Dits et Écrits, vol. III, texte $n^{\circ} 192$, cité par J. Revel, Le Vocabulaire de Foucault, Paris, Ellipses, 2002) : « On admet que le structuralisme a été l'effort le plus systématique pour évacuer non seulement de l'ethnologie, mais de toute une série d'autres sciences, et même à la limite de l'histoire, le concept d'événement. Je ne vois pas qui peut être plus anti-structuraliste que moi ». 
3. Dans Lyotard (1985: 286), les figures sont définies comme la «violation de l'ordre du discours", violations signifiant qu'un autre espace que l'espace linguistique s'insinue dans le discours, «qu'il y produit des effets de sens qui ne peuvent résulter du jeu normal des données sémantiques et/ou syntaxiques, mais procèdent de leur transgression. Cette transgression suppose qu'une force agit dans l'espace linguistique ».

4. La figure est conçue comme une force agissant, mais cette action a lieu «dans l'espace linguistique » (ibid.).

5. Voir Zourabichvili $(2003: 37)$ : le langage a pour rôle d'effectuer la «synthèse disjonctive » de l'événement.

6. Voir Lyotard (1985: 13) : «La position de l'art est un démenti à la position du discours. La position de l'art indique une fonction de la figure, qui n'est pas signifiée, et cette fonction autour et jusque dans le discours. Elle indique que la transcendance du symbole est la figure, c'est-à-dire une manifestation spatiale que l'espace linguistique ne peut pas incorporer sans être ébranlé, une extériorité qu'il ne peut pas intérioriser en signification. " Ainsi, l'événement que constitue la figure se situe à la fois dans le langage, inscrit dans la matérialité de celui-ci, tout en ne pouvant être réductible à la signification, c'est-à-dire au mode de fonctionnement imposé par le système de la langue.

7. Ainsi, Foucault ne veut pas accepter de «dehors de l'histoire : on ne sort pas de son propre temps; ou, plus exactement, on ne sort pas du temps tout court : on est toujours, à un titre ou à un autre, plongé dans l'histoire.» (Revel 2010 : 96).

8. Il y a, selon Lyotard, une première violence, qui est celle de la catégorisation, de la transformation en langage de la chose, puis une autre, qui lui est inverse, op. cit., p. 14 : «cette violence fait de l'objet un signe, mais elle fait symétriquement du discours une chose, elle met de l'épaisseur, elle dresse une scène dans l'articulation et dans la limpidité de la signification ».

9. Voir Lyotard (1971 : 146) : «Si j'appelle l'énoncé je te musique une figure, il faut dire que cette figure (et mon hypothèse est : que toute figure) est chargée linguistiquement, c'est-à-dire fait événement linguistique, parce qu'elle est un effet de décharge provenant d'un autre ordre ».

10. Voir Lyotard (1971: 135) : «La vérité ne se trouve pas dans l'ordre de la connaissance, elle se rencontre dans son désordre, comme événement. ", ou Lyotard (1971: 282) : "Voici pourtant notre hypothèse : on peut échapper à cette alternative de l'espace figural qui trompe et de l'espace textuel où se constitue la connaissance. En-deçà de cette alternative, on peut discerner une autre fonction, qui y est omise, et qui serait articulée par principe sur l'espace figural, une fonction de vérité ».

11. Lyotard situe ainsi son approche à la croisée de la linguistique, de la logique (fregienne), de la philosophie et de l'esthétique : voir Gualandi $2017: 280$.

12. Voir Vallespir (2013: 93): "Cette énaction du sujet par le texte et du texte par le sujet suppose que toutes les dimensions déterminantes du sujet (ensemble virtuellement infini : biologiques, sensori-motrices, psychologiques, sociales et culturelles, mas aussi contexte de la détermination du présent de la lecture) y soient impliquées au même titre, de même que toutes les dimensions déterminant le texte (dont l'époque, le genre, le sous-genre, le type). Au-delà, la niche sémiotique que constitue l'acte de lecture ne peut être conçue qu'à partir d'une infinité d'autres niches sémiotiques qu'elle présuppose : niches tenant aux couplages du sujet à d'autres textes, plus largement à d'autres supports sémiotiques, enfin à d'autres types d'expériences plus centralement motrices par exemple. A l'inverse, l'énaction d'un texte suppose virtuellement l'énaction de ce texte par d'autres sujets. C'est ainsi ce réseau virtuellement infini de niches, d'énactions qui définit la lecture du texte comme énaction. »

13. Nous nous livrons une fois encore à la description de la notion de multistabilité bien qu'elle ait été déjà présentée dans les articles cités, avec les redites que cela implique ainsi que la reprise de certaines citations, à des fins de clarté dans l'exposition. Nous prions le lecteur qui les aurait lues dans un autre article de bien vouloir nous en excuser. 
14. «L'occultation qui accompagne l'énoncé Je te musique n'est plus l'effacement du système dans le clair-obscur (ou le préconscient) grâce auquel se soutient « je te connais » en pleine lumière. Le retrait des éléments absents ne crée pas de la virtualité, mais de la violence. Musique est un terme actualisé par transgression, sa présence témoigne qu'il y a en sous-sol, non pas un système, mais des forces, une énergétique qui bouscule l'ordonnancement du système. Quand vous faites un verbe avec un substantif, il y a événement [...]», p. 145. L'analyse de Lyotard a pour vocation de mettre en valeur le fonctionnement non structural du langage, et souligner l'irruption d'un autre ordre dans le langage, inconscient, force, énergie, par la « violence » qui s'y joue.

15. Ainsi, dans les répliques précédentes entre les deux personnages intervenant à ce moment du dialogue, A (l'amoureuse) et $\mathrm{P}$ (le poète), non citées par Lyotard, « je te musique » est pris dans une série répondant à la même structure $[\mathrm{je}+\mathrm{te}+$ verbe d'action] : «P. je t'imprime/ A. je te savoure/ P. je te rame/ A. je te précède », (Pichette 1969: 56). "Je te musique " est lui-même associé à un de ces verbes ((la citation réelle étant «P. je t’innerve te musique »), quand la substitution d'un nom dans ce paradigme a déjà été proposée dans le couple de répliques précédent («P. je te vertige /A. et tu me recommences »).

16. Épreuve aux sels d'argent rehaussée de crayon et encre de Chine, 28.2 x $22.5 \mathrm{~cm}, 1924$.

17. Deleuze et Guattari continuent ainsi : «Bateson cite en exemple la culture balinaise, où des jeux sexuels mère-enfant, ou bien des querelles entre hommes, passent par cette bizarre stabilisation intensive. "Une espèce de plateau continu d'intensité est substitué à l'orgasme”, à la guerre ou au point culminant » (Deleuze \& Guattari 1980 : 32).

18. En réalité, même si la métaphore est donnée pour stable dans la doxa analytique, qui a refermé essentiellement sur la propriété de «courage » le métaphorisé du tour, n'en demeure pas moins un certain halo d'instabilité. Toute métaphore est donc par nature instable, mais à des degrés divers (Vallespir 2017b : 490-491).

19. Voir à cet égard les analyses de J.-M. Klinkenberg, qui propose de voir dans la métaphore la figure « qui procède le plus nettement à la recatégorisation de l'expérience » (Klinkenberg 2000 : 366).

20. Plus exactement, G. Molinié pose la catégorie de réception d'archive, qu'il oppose à la réception esthétique, réception littérarisée. La lecture d'archive est définie comme «lecture documentaire, [...] archivistique, [...] historique, [...] philologique » (Molinié $1998: 136$ ).

\section{ABSTRACTS}

Dans cet article, on tente de poser ce que peut être un événement stylistique conçu comme " plateau multistable», en convoquant les philosophies des années 1970 en France et l'approche neurophénoménologique de Varela pour les articuler ensemble. Après avoir circonscrit les caractéristiques de l'événement selon Deleuze, Foucault et Lyotard, on met en évidence la parenté de ce concept avec la notion varélienne d'énaction. On tente enfin de donner à l'événement, qui, en intension, répond à la définition donnée par cette philosophie française, une extension possible: celle de "plateau multistable». À cette fin, on choisit de remettre sur le métier une figure choisie par Lyotard pour exemplifier sa définition de l'événement figural, «je te musique ", et de l'analyser dans une nouvelle perspective, à l'aune de l'approche énactive de F. Varela. On montre ainsi que cette figure donne lieu à un agencement de "plateau multistable ", lequel, par ses propres prérogatives et dans ce nouveau cadre, est à son tour propre à faire événement au sein du texte. 
INDEX

Chronological index: XXe siècle

Mots-clés: événement stylistique, énaction, instabilité, poésie, métaphore

\section{AUTHOR}

\section{MATHILDE VALLESPIR}

Sorbonne Université, EA STIH

Mathilde Vallespir est maîtresse de conférences à la faculté de lettres de Sorbonne université en sémiotique littéraire comparée. Elle a publié un ouvrage, Lire, écouter, exorciser la guerre. Essai de sémiotique comparée (poésie/musique) (Paris, Champion, 2012) et dirigé plusieurs ouvrages collectifs dont, avec L. Kurts-Woeste et M.-A. Watine, La Violence du logos : entre sciences du texte, philosophie et littérature (Paris, Garnier, 2013) et avec D. Maingueneau, Lire Derrida? Autour d'Eperons. Les styles de Nietzsche (Paris, Lambert Lucas, 2015). 